\title{
SOME THEOREMS ON GROUPS WITH APPLICATIONS TO RING THEORY
}

\author{
BY \\ BAILEY BROWN AND NEAL H. MCCOY
}

1. Introduction. This paper is an outgrowth of an attempt to give a unified treatment of certain results in the theory of rings. For example, in a recent paper [5] $\left(^{1}\right)$ it was shown that the set of all elements of a ring $R$ which generate regular ideals $\left({ }^{2}\right)$ is itself an ideal in $R$. In that paper it was observed that the proof of this result is in many ways analogous to the proof in Brown and McCoy [4] that the radical of $R$, as defined in [3], is an ideal. In $\$ 2$ of the present paper a theorem will be proved in the general setting of group theory which yields as special cases both of these results as well as a number of other cases of some interest. Some notation needs to be introduced before stating this theorem.

Let $G$ be a group additively written, although $G$ is not required to be abelian; and let $\Omega$ be a fixed set of endomorphisms of $G$ which includes all inner automorphisms. The $\Omega$-subgroup of $G$ generated by an element $b$ may be denoted by $(b)$. It is assumed that a mapping $F$ is given which associates with each element $a$ in $G$ a unique subgroup $F(a)$ of $G$ in such a way that if $a, b \in G$, then

$\mathrm{P}_{1}$.

$$
\begin{aligned}
& F(a+b) \subseteq F(a)+(b), \\
& b \in F(a) \text { implies that } F(a+b) \subseteq F(a) .
\end{aligned}
$$

$\mathrm{P}_{2}$.

Under these conditions, $G$ may be called an $(F, \Omega)$-group.

Note that $F(a)$ is not required to be an $\Omega$-subgroup, but if it happens to be an $\Omega$-subgroup, $\mathrm{P}_{2}$ is implied by $\mathrm{P}_{1}$.

An element $a$ of $G$ may be said to be $F$-regular if and only if $a \in F(a)$, and a subset of $G$ is called $F$-regular if and only if each of its elements is $F$-regular. The theorem mentioned above (Theorem 1 ) states that if $G$ is an $(F, \Omega)$-group, the set $N=\{a \in G ;(a)$ is F-regular $\}$ is an F-regular $\Omega$-subgroup of $G$ which contains every $F$-regular $\Omega$-subgroup of $G$.

In $\$ 3$ it is shown how to make any $\Omega$-homomorphic image of $G$ into an $(F, \Omega)$-group and then it is proved in Theorem 3 that the $\Omega$-group $G-N$ has no nonzero $F$-regular $\Omega$-subgroup.

In some of the applications to rings it will not be necessary to require the

Presented to the Society, April 28, 1950; received by the editors February 27, 1950 and, in revised form, April 7, 1950.

(1) Numbers in brackets refer to the bibliography at the end of the paper.

(2) The word regular is being used in the von Neumann sense, that is, an element $a$ is regular if and onty if there is an element $x$ such that $a x a=a$, and an ideal is regular if and only if each element of the ideal is regular. 
associative law of multiplication, and thus some of our results are obtained for not necessarily associative ring or narings. Accordingly, $\$ 4$ is devoted to a discussion of the concept of regularity in a naring, and certain results are proved which generalize some of those established in [5] for rings. In $\$ 5$ an application of Theorem 1 is obtained for the case in which the naring is specialized to an alternative naring. In the following section there are given a number of other illustrations of the application of Theorem 1 to rings.

Starting with $\$ 7$ the assumptions on $F$ are strengthened, and it is then possible to express $N$ as the intersection of a certain class of $\Omega$-subgroups of $G$. Inasmuch as a precise statement of this and other related results requires some additional notation, a detailed account will be postponed until later. The treatment is based on recent work of Brown [2] and in turn generalizes his results. Special cases of our Theorems 6 and 7 give at least an important part of the theory of each of the following: the radical or, more generally, the F-radical of a ring [3]; the radical (F-radical) of a naring according to Smiley [10]; the Jacobson radical of a ring [7]; the Jacobson radical of a naring or cluster as presented by Brown [2]. Thus, for the first time, these diverse theories are here subsumed under one general theory.

In the Jacobson theory the concept of primitive ring plays an important role, while subdirectly irreducible rings of zero F-radical enter naturally in to the theory of the $F$-radical. Previous attempts to unify these theories have failed at least partly because of the difficulty of obtaining an appropriate generalization of these quite different concepts. Such a generalization is exhibited in $\S 8$, and the rest of the theory then follows by an easy extension of the work of Brown [2].

The referee has pointed out that Theorems $1-3,6$, and 7 make no essential use of the associativity of addition, and that our proofs (except for that of Theorem 1 which can be suitably rephrased) are valid in the more general setting of a loop with operators. We are also indebted to the referee for suggestions which have improved our proof of Theorem 5 as well as the discussion of $\S 5$.

2. Existence of greatest $F$-regular $\Omega$-subgroup. Let $G$ be an $(F, \Omega)$-group as defined in the introduction. Since $\Omega$ is assumed to contain all inner automorphisms, an $\Omega$-subgroup of $G$ is necessarily a normal subgroup. It follows readily that $(a+b) \subseteq(a)+(b)$, a fact which will be used presently.

We may now prove the following theorem which is fundamental for our purposes:

Theorem 1. If $G$ is an $(F, \Omega)$-group, the set $N=\{a \in G ;(a)$ is F-regular $\}$ is an $F$-regular $\Omega$-subgroup of $G$ which contains every F-regular $\Omega$-subgroup of $G$.

If $z, w \in N$, we show first that $(z-w)$ is $F$-regular. If $a \in(z-w)$, the observation made above shows that $a=u-v$ for some $u$ in $(z), v$ in (w). But $u=a+v$ is $F$-regular since $z \in N$, so $a+v \in F(a+v) \subseteq F(a)+(v)$ by $\mathrm{P}_{1}$. Thus we 
may write $a+v=-b+v_{1}$ for some $b$ in $F(a)$ and $v_{1}$ in $(v)$. It follows that $a=-b+v_{1}-v=v_{2}-b$ for suitable $v_{2}$ in the normal subgroup $(v)$. Hence $a+b \in(v) \subseteq(w)$. Since $w \in N, a+b \in F(a+b) \subseteq F(a)$, by $\mathrm{P}_{2}$ since $b \in F(a)$. Since $F(a)$ is a group, $a \in F(a)$. Thus $(z-w)$ is $F$-regular, and $z-w \in N$. If $\omega \in \Omega$, then $z \omega \in(z),(z \omega) \subseteq(z)$; thus $z \omega \in N$. This shows that $N$ is an $\Omega$-subgroup of $G$, and it is certainly $F$-regular. If $H$ is an $F$-regular $\Omega$-subgroup of $G$, and $b \in H$, then $(b) \subseteq H,(b)$ is $F$-regular. Hence $b \in N$, so $H \subseteq N$. This completes the proof of the theorem.

In view of the properties just proved, $N$ may be called the greatest F-regular $\Omega$-subgroup of $G$.

Before proceeding, we may illustrate this theorem by a few simple examples. First, let $G$ be the additive group of a ring $R$, and let $\Omega$ be the identity automorphism together with the set of all right and left multiplications by elements of $R$. Thus an $\Omega$-subgroup is just an ideal in $R$. Let us set

$$
F(a)=\left\{a r-r+\sum\left(x_{i} a y_{i}-x_{i} y_{i}\right)\right\}
$$

where $r, x_{i}, y_{i}$ range over $R$ and the sums are finite. It is easily verified that properties $\mathrm{P}_{1}$ and $\mathrm{P}_{2}$ hold, and thus $G$ is an $(F, \Omega)$-group. In this case, $N$ is the radical of $R$ as defined in [3], and Theorem 1 furnishes an elementary proof, closely related to that in [4], that the radical is an ideal.

As a next example, let $G$ and $\Omega$ be as in the preceding example but define $F(a)=\{a x-x ; x \in R\}$. It then follows that $N$ is the Jacobson radical of $R$. If we let $R$ be an arbitrary naring (or cluster [6]), and take $F(a)$ to be the right ideal generated by the set $\{a x-x ; x \in R\}$, Theorem 1 shows that $N$ is an ideal which naturally has been called the Jacobson radical of the naring (or cluster) [2].

Again, with the same definition of $G$ and $\Omega, R$ now being a ring, let $F(a)$ $=a R a$. Thus $a \in F(a)$ if and only if $a$ is regular, and Theorem 1 asserts that that there exists a greatest regular ideal in an arbitrary ring [5].

Additional applications of this theorem will be given after $\$ 3$.

3 . Extension to $\Omega$-homomorphic images. We return to the general case in which $G$ is an arbitrary $(F, \Omega)$-group. If $K$ is an $\Omega$-subgroup of $G$, then it is well known that $G-K$ is an $\Omega$-group under the natural definition $(a+K) \omega$ $=a \omega+K$. Conversely, any $\Omega$-homomorph $G^{*}$ of $G$ is $\Omega$-isomorphic to $G-K$, where $K$ is the $\Omega$-subgroup of $G$ which is the kernel of the homomorphism. The following theorem gives a natural way to make $G^{*}$ into an $(F, \Omega)$-group, the $\Omega$-homomorphic image of $F(a)$ being denoted by $F(a)^{*}$ :

THEOREM 2. If $a \rightarrow a^{*}$ is an $\Omega$-homomorphism of an $(F, \Omega)$-group $G$ onto an $\Omega$-group $G^{*}$ and we set $F\left(a^{*}\right)=F(a)^{*}$, then $F\left(a^{*}\right)$ is well defined and under this definition $G^{*}$ becomes an $(F, \Omega)$-group.

First we show that $F\left(a^{*}\right)$ is well defined, that is if $a^{*}=b^{*}$, then $F(a)^{*}$ $=F(b)^{*}$. Let $K$ be the kernel of the $\Omega$-homomorphism of $G$ onto $G^{*}$, so that 
$a^{*}=b^{*}$ implies that $a=b+k$ for some $k$ in $K$. Then by $\mathrm{P}_{1}, F(a)=F(b+k)$ $\subseteq F(b)+(k)$, and hence $F(a)^{*} \subseteq F(b)^{*}$. In like manner, it follows that $F(b)^{*}$ $\subseteq F(a)^{*}$, and thus we must have $F(a)^{*}=F(b)^{*}$.

The following calculation now shows that $\mathrm{P}_{1}$ is satisfied in $G^{*}$ :

$$
F\left(a^{*}+b^{*}\right)=F\left((a+b)^{*}\right)=F(a+b)^{*} \subseteq(F(a)+(b))^{*}=F(a)^{*}+\left(b^{*}\right) . .
$$

To prove $\mathrm{P}_{2}$ we observe that if $b^{*} \in F\left(a^{*}\right)=F(a)^{*}$, then $b-c \in K$ for some $c$ in $F(a)$. Thus

$$
F\left(a^{*}+b^{*}\right)=F\left(a^{*}+c^{*}\right)=F\left((a+c)^{*}\right)=F(a+c)^{*} \subseteq F(a)^{*}=F\left(a^{*}\right),
$$

and the proof of the theorem is completed.

For the moment, let us denote the $N$ of Theorem 1 by $N(G)$ to emphasize the particular $(F, \Omega)$-group $G$ being considered. Now if $G^{*}$ is any $\Omega$-homomorph of $G$, the preceding theorem shows that $N\left(G^{*}\right)$ has meaning. We may now prove the following result:

THEOREM 3. If $G$ is an $(F, \Omega)$-group, the greatest $F$-regular $\Omega$-subgroup of $G-N(G)$ is the zero subgroup, that is, $N(G-N(G))=0$.

Let $a^{*}$ denote the coset $a+N(G)$. If $b^{*} \in N(G-N(G))$ and $a \in(b)$, then $a^{*} \in\left(b^{*}\right)$. Hence $a^{*} \in F\left(a^{*}\right)=F(a)^{*}$, so $a+c \in N(G)$ for some element $c$ in $F(a)$. It follows that $a+c \in F(a+c) \subseteq F(a)$ since $c \in F(a)$. Hence $a \in F(a)$, $b \in N(G), b^{*}=0$, and the proof is completed.

It is easy to apply this theorem to the examples given above in which $G$ is the additive group of a ring $R$, and $\Omega$ consists of the identity automorphism together with all right and left multiplications. In this case $N$ is an ideal in $R$, and by a natural definition of multiplication in the $\Omega$-group $G-N$, it becomes the residue class ring $R / N$. Furthermore, an $\Omega$-subgroup of the additive group of $R / N$ is just an ideal in $R / N$. Thus, for example, in the case of the first illustration given above, Theorem 3 merely states that if $N$ is the radical of $R$, then the residue class ring $R / N$ has zero radical.

We showed in Theorem 2 that if $G$ is an $(F, \Omega)$-group, then there is a natural way to make any $\Omega$-homomorph $G^{*}$ of $G$ into an $(F, \Omega)$-group. The following theorem shows that in a certain sense this procedure can sometimes be reversed:

THEOREM 4. If $a \rightarrow F(a)$ is a mapping which associates with each element a of the $\Omega$-group $G$ a unique $\Omega$-subgroup $F(a)$ of $G$ with the property that, in each $\Omega$-homomorph $G^{*}$ of $G, a^{*}=b^{*}$ implies that $F(a)^{*}=F(b)^{*}$, then $G$ is an $(F, \Omega)$ group.

It may be emphasized that heretofore $F(a)$ has been required merely to be a subgroup of $G$, but a part of the hypothesis of this theorem is that it be an $\Omega$-subgroup. To reach the desired conclusion we need only show that in $G$, $F(a)$ has properties $\mathrm{P}_{1}$ and $\mathrm{P}_{2}$. Accordingly, let $a$ and $b$ be elements of $G$, 
and let $G^{*}$ be the $\Omega$-homomorph $G-(b)$ of $G$. Since $(a+b)^{*}=a^{*}$, one of our hypotheses implies that $F(a+b)^{*}=F(a)^{*}$. Thus $F(a+b) \subseteq F(a)+(b)$, which is just property $\mathrm{P}_{1}$. Property $\mathrm{P}_{2}$ then follows immediately since $F(a)$ is here an $\Omega$-subgroup.

Before proceeding to further applications, we may observe that this theorem makes it possible to apply Theorems 1 and 3 to the $F$-radical of a ring as developed in [3]. This will be mentioned again in $\S 6$.

4. Regularity in a naring. Let $G$ be the additive group of an arbitrary naring $R$, and let $\Omega$ be the set of all right and left multiplications together with the identity automorphism. If $c \in R, c$ induces a right multiplication $\rho(c)$ and a left multiplication $\lambda(c)$. Let $\mathrm{P}$ denote a finite product of one or more right multiplications, and $\Lambda$ a finite product of one or more left multiplications.

If $a \in R$, let $F(a)$ denote the set of all finite sums $\sum_{k} a \mathrm{P}_{k} \cdot a \Lambda_{k}$. For example $(a x)(y a)+(((a r) s) t)(u a)$ is in $F(a)$. Since, by definition, each $\mathrm{P}_{k}\left(\Lambda_{k}\right)$ must contain at least one right (left) multiplication, an expression such as (ax)a $+a a$ need not be in $F(a)$. However, if $a=(a x) a+a a$, substitution of the entire right member for certain of the elements $a$ appearing in the right member shows that $a \in F(a)$. We shall say that an element $a$ is regular if and only if $a \in F(a)$, while a subset of $R$ is regular if and only if each of its elements is regular. If $R$ happens to be associative, it is clear that the present definition of regularity coincides with the well known one of von Neumann, except that we do not require a unit element.

It is easy to verify that $F(a)$ is a subgroup of $G$ satisfying conditions $\mathrm{P}_{1}$ and $\mathrm{P}_{2}$, and hence $G$ is an $(F, \Omega)$-group. Thus Theorems 1 and 3 show the existence of a greatest regular ideal $M(R)$ in $R$, and that $M(R / M(R))=0$. These are generalizations of results obtained in [5] for the case in which $R$ happens to be associative. We shall now indicate a proof of the following theorem, which is well known if $R$ is a ring.

THEOREM 5. If $R_{n}$ is the complete matrix naring of order $n$ over a naring $R$, then $R_{n}$ is regular if and only if $R$ is regular.

It is almost obvious that $R$ is regular if $R_{n}$ is regular, and we shall accordingly confine our remarks to the other part of the theorem. The proof will be based on the observation that if $a-c$ is regular and $c \in F(a)$, then $a$ is regular.

If $u \in R$, let $U_{i j}$ denote the element of $R_{n}$ with $(i, j)$ entry $u$ and zeros elsewhere. Supposing $R$ regular, let $B$ be an element of $R_{n}$ with $(i, j)$ entry $b_{i j}$. Since $b_{i j}$ is regular, we may write $b_{i j}=\sum_{k} b_{i j} \mathbf{P}_{i j k} \cdot b_{i j} \Lambda_{i j k}$. We associate with the element $b_{i j}$ an element $\sum_{k} B \mathrm{P}_{i j k}^{\prime} \cdot B \Lambda_{i j k}^{\prime}$ of $F(B)$ in $R_{n}$, where the first factor of each $\mathrm{P}^{\prime}$ is obtained from the first factor $\rho(x)$ of the corresponding $\mathrm{P}$ by replacing $x$ by $X_{j i}$, and the remaining factors of each $\mathrm{P}^{\prime}$ and all factors of each $\Lambda^{\prime}$ are obtained from the corresponding factors $\rho(y)$ of $\mathrm{P}$ and $\lambda(z)$ of $\Lambda$ by replacing $y$ by $Y_{i i}$ and $z$ by $Z_{i i}$. For example, if $b=b_{i j}$ and $b=(b x)(y(z b))$ 
$+(((b s) t) u)(v b)$, the associated element of $F(B)$ is $\left(B X_{j i}\right)\left(Y_{i i}\left(Z_{i i} B\right)\right)$ $+\left(\left(\left(B S_{j i}\right) T_{i i}\right) U_{i i}\right)\left(V_{i i} B\right)$. We now define $B^{i j}=B-\sum_{k} B \mathrm{P}_{i j k}^{\prime} \cdot B \Lambda_{i j k}^{\prime}$. It follows from the above italicized observation that if some $B^{i j}$ is regular, then $B$ is regular. If $b_{r s}^{i j}$ is the $(r, s)$ entry of $B^{i j}$, it may be verified that

$$
b_{r s}^{i j}=b_{r s}-\sum_{k} b_{r j} \mathrm{P}_{i j k} \cdot b_{i s} \Lambda_{i j k}
$$

Clearly $b_{i j}^{i j}=0$ by the equation defining the regularity of $b_{i j}$. Moreover $b_{r s}^{i j}=0$ if $b_{r s}=b_{r j}=0$ or if $b_{r s}=b_{i s}=0$.

It now follows from (1) that if $A \in R_{n}$, then $A_{1}=A^{11}$ has its $(1,1)$ entry zero, and that $A_{2}=A_{1}^{12}$ has its $(1,1)$ and $(1,2)$ entries zero. Proceeding to introduce zeros elementwise by rows, and setting $m=n^{2}$, we see finally that $A_{m}=A_{m-1}^{n n}$ is the zero matrix, which is regular. This implies that $A_{m-1}$ is regular, and after $n^{2}$ backward steps we infer that $A$ is regular, completing the proof.

Actually, a somewhat more precise theorem can be proved in that Theorem 4 of [5] can be completely extended to the naring case. However, the complexity of the notation makes it probably not worth while to write out the details of the proof.

5. Regularity in an alternative naring. We now assume that $R$ is an alternative naring, namely, that $(a b) c-a(b c)$ is an alternating function of its arguments $a, b, c$. Thus $(a x) a=a(x a)$, and we may denote this element by $a x a$ without ambiguity. Let $G$ and $\Omega$ be as in the preceding section, but define $F(a)=a R a$. Following Smiley [9], we shall say that $a$ is regular if and only if $a \in F(a)$. It is easy to verify $\mathrm{P}_{1}$ and we shall show that $\mathrm{P}_{2}$ holds. This is done by means of the following identities valid in any alternative $\operatorname{ring}\left({ }^{3}\right)$ :

$$
\begin{aligned}
(a x)(y a) & =a(x y) a, \\
(a x a) y & =a(x(a y)) .
\end{aligned}
$$

We must show that $(a+a s a) t(a+a s a)=((a+a s a) t)(a+a s a)$ is in $F(a)$ for all $s, t$ in $R$. After applying the distributive laws, it may be verified as follows that each of the four summands is in $F(a)$. Using (2), (at) (asa) $=a(t(a s)) a$. By (3), ((asa)t)a=a(s(at))a. Finally, (3) shows that $((a s a) t)(a s a)$ $=(a(s(a t)))((a s) a)$ which is in $F(a)$ by (2). Hence $\mathrm{P}_{2}$ is satisfied.

It follows that $G$ is an $(F, \Omega)$-group, and Theorems 1 and 3 show the existence of a greatest regular ideal $N(R)$ in $R$ and that $N(R / N(R))=0$.

If $R$ is alternative, not only is $N(R)$ defined, but also the $M(R)$ of the preceding section. Obviously $N(R) \subseteq M(R)$, but the exact relation between them is an unsolved problem.

One other remark may be in order. It may be shown that $R_{n}(n>1)$ is 12].

(3) Apparently (2) and (3) were first proved by Moufang [8] from an identity of Zorn [11, 
alternative only if $R$ is associative. However, if $R$ is alternative, $R_{n}$ is still a regular naring in the sense of $\S 4$ whenever $R$ is regular under either definition.

6. Additional applications to rings. Throughout this section $R$ will be an arbitrary (associative) ring, and in our applications it is to be understood that $G$ is the additive group of $R$ and $\Omega$ the identity automorphism together with all right and left multiplications by elements of $R$. We shall, for the most part, confine ourselves to giving various definitions of $F(a)$ which satisfy $\mathrm{P}_{1}$ and $\mathrm{P}_{2}$ and therefore make $G$ into an $(F, \Omega)$-group. The actual verification of these properties will be left to the reader.

EXAMPLE 1. Set $F(a)=a^{2} R$, and hence $a \in F(a)$ if and only if $a$ is strongly regular as defined by Arens and Kaplansky [1]. The $N$ of our Theorem 1 is then the greatest strongly regular ideal in $R$.

ExAmple 2. We now set $F(a)=a(a)$. In this case, $a \in F(a)$ if and only if $a=a a_{1}$, where $a_{1} \in(a)$. We shall say that $a$ is weakly regular if and only if $a \in F(a)$, and Theorem 1 states the existence of a greatest weakly regular ideal in $R$.

We pause to make a few remarks about weak regularity. In the first place, it is clear that if $a$ is regular, it is weakly regular. Following Arens and Kaplansky [1] let us call an element $a$ biregular if and only if $(a)=(e)$, where $e$ is a central idempotent. If $a$ is biregular, then for some integer $n$ and some element $r$ or $R, a=n e+e r$, where $e$ is a central idempotent. Thus $a e=a$ with $e \in(a)$, and hence $a$ is weakly regular. This shows that biregularity of an element $a$ implies that $a$ is weakly regular. On the other hand, examples given by Arens and Kaplansky show that regularity neither implies nor is implied by biregularity, so weak regularity coincides with neither. Moreover, weak regularity of every element of a ring implies that the ring is semi-simple in the sense of Jacobson.

Further discussion of this concept would take us too far afield, but we may remark that if in the statement of each theorem in [5] the word "regular" is replaced by "weakly regular," the resulting theorem is true. The proofs follow the same general pattern as those of the corresponding theorem in [5] but naturally involve somewhat longer and more involved calculations.

ExAmple 3. Let $F(a)$ be an ideal, defined for every ring, and such that in any homomorph $R^{*}$ of $R, F\left(a^{*}\right)=F(a)^{*}$. Theorem 4 then shows that $G$ is an $(F, \Omega)$-group, and the $N$ of Theorem 1 is in this case the $F$-radical of $R[3]$. Actually, the associativity of multiplication plays no role here as was pointed out by Smiley [10] in extending these results to narings.

Example 4. Define $F(a)$ to be the set of all polynomial expressions of the form $n_{2} a^{2}+n_{3} a^{3}+\cdots$, where the $n_{i}$ are integers. It is easy to verify that $a \in F(a)$ if and only if $a x a=a, x$ a polynomial in $a$ with integral coefficients. Thus $a \in F(a)$ implies that $a$ is regular in the subring of $R$ generated by $a$.

ExAmple 5. Let $F(a)$ be the set of all elements of $R$ of the form $a y-y$, where $y$ is a polynomial expression of the form $n_{1} a+n_{2} a^{2}+\cdots$, the $n_{i}$ being 
integers. The greatest $F$-regular ideal in $R$ is certainly contained in the Jacobson radical of $R$, and coincides with it in any ring in which the Jacobson radical is a nil ideal. This follows at once from the formula which shows that a nilpotent element is necessarily $F$-regular $[7$, p. 304]. In general, however, the greatest $F$-regular ideal in $R$ is properly contained in the Jacobson radical.

7. Definition of $\left(F, \Omega_{1}, \Omega\right)$-group and related concepts. In order to give a unified account of various theories of the radical of a ring we need to strengthen our requirements $\mathrm{P}_{1}$ and $\mathrm{P}_{2}$ on the mapping $F$. Let $G$ be an arbitrary group, additively written, $A$ the group of inner automorphisms of $G$, $\Omega_{1}$ and $\Omega$ fixed sets of endomorphisms of $G$ such that $A \subseteq \Omega_{1} \subseteq \Omega$. By $(a)$, we shall means as before the $\Omega$-subgroup of $G$ generated by $a$, and $(a)_{1}$ will denote the $\Omega_{1}$-subgroup of $G$ generated by $a$. We now, and henceforth, assume that there exists a mapping $F$ which associates with each element $a$ in $G$ a unique $\Omega_{1}$-subgroup $F(a)$ in such a way that for all $a, b$ in $G$,

P.

$$
F(a+b) \subseteq F(a)+(b)_{1} .
$$

Under these conditions we shall call $G$ an $\left(F, \Omega_{1}, \Omega\right)$-group.

We may observe that since $F(a)$ is now required to be an $\Omega_{1}$-subgroup, $b \in F(a)$ implies that $(b)_{1} \subseteq F(a)$, and hence properties $\mathrm{P}_{1}$ and $\mathrm{P}_{2}$ are certainly satisfied. Thus an $\left(F, \Omega_{1}, \Omega\right)$-group is an $(F, \Omega)$-group, and Theorem 1 is immediately at our disposal. Furthermore, it is easy to show that the definition of $F\left(a^{*}\right)$ used in Theorem 2 makes any $\Omega$-homomorph $G^{*}$ of the $\left(F, \Omega_{1}, \Omega\right)$ group $G$ into an $\left(F, \Omega_{1}, \Omega\right)$-group, and so the results of $\S 3$ likewise remain valid for $\left(F, \Omega_{1}, \Omega\right)$-groups.

An $\Omega_{1}$-subgroup $I$ of the $\left(F, \Omega_{1}, \Omega\right)$-group $G$ is said to be modular if and only if there exists an element $e$ not in $I$ such that $F(e) \subseteq I$. A modular $\Omega_{1}$-subgroup $I$ is large if and only if there exists an element $e$ not in $I$ such that $F(e) \subseteq I$, with the additional requirement that $e$ is contained in every $\Omega_{1}$-subgroup which properly contains $I$. By use of Zorn's lemma, any modular $\Omega_{1}$-subgroup of $G$ can be extended to a large modular $\Omega_{1}$-subgroup which does not contain $e$.

8. Intersection theory and subdirect sums. If $I$ is any subgroup of $G$, let us set $I^{\prime}=\{a \in G ;(a) \subseteq I\}$, that is, $I^{\prime}$ is the greatest $\Omega$-subgroup of $G$ contained in $I$. This notation will be used consistently henceforth.

We may now prove the following result:

THEOREM 6. If $G$ is an $\left(F, \Omega_{1}, \Omega\right)$-group, the set $N=\{a \in G ;(a)$ is F-regular $\}$ is the intersection $X$ of all $\Omega$-subgroups $M^{\prime}$ where $M$ is a large modular $\Omega_{1}$-subgroup of $G$.

We show first that $X \subseteq N$. If $a \notin N$, then $b \notin F(b)$ for some $b$ in $(a)$. Then $F(b)$ is modular, and use of Zorn's lemma yields a large modular $\Omega_{1}$-subgroup $M$ such that $b \notin M$. Since $b \in(a)$, it follows that $a \notin M^{\prime}$; so $a \notin X$. 
Conversely, $N \subseteq X$. For if $a \notin X$, there exists a large modular $\Omega_{1}$-subgroup $M$ such that $a \notin M^{\prime}$. Hence $b \notin M$ for some $b$ in $(a)$. Now there exists an element $e$ not in $M$ such that $F(e) \subseteq M$ and such that any $\Omega_{1}$-subgroup which properly contains $M$ must contain $e$ also. Thus $e \in(b, M)_{1}$, that is, $e=c+m$ for some $c$ in $(b)_{1}$ and $m$ in $M$. Suppose that $a \in N$. Then $c \in(b)_{1} \subseteq(b) \subseteq(a)$, so $c \in F(c)$. Hence, by property $\mathrm{P}, e-m \in F(e-m) \subseteq F(e)+(m)_{1} \subseteq M$, so $e \in M$, a contradiction. Hence $a \in N$, and the proof is completed.

An $\left(F, \Omega_{1}, \Omega\right)$-group may be said to be primitive if and only if it contains a large modular $\Omega_{1}$-subgroup $M$ such that $M^{\prime}=0$. It follows from the preceding theorem that if $G$ is primitive, then $N=0$. We shall next prove the following lemma:

Lemma. If $M$ is a large modular $\Omega_{1}$-subgroup of $G$, then the $\left(F, \Omega_{1}, \Omega\right)$ group $G-M^{\prime}$ is primitive.

Denoting natural $\Omega$-homomorphic images in $G^{*}=G-M^{\prime}$ by asterisks, we first show that $M^{*}$ is a large modular $\Omega_{1}$-subgroup of $G^{*}$. Let $e$ be an element not in $M$ such that $F(e) \subseteq M$, and such that any $\Omega_{1}$-subgroup of $G$ which properly contains $M$ also contains $e$. Then $F\left(e^{*}\right)=F(e)^{*} \subseteq M^{*}$. If $e^{*} \in M^{*}$, then $e \in M+M^{\prime}=M$, so $e^{*} \notin M^{*}$. This shows that $M^{*}$ is modular. Now let $M_{1}^{*}$ be any $\Omega_{1}$-subgroup of $G$ which properly contains $M^{*}$. Then $M_{1}^{*}$ is the image of an $\Omega_{1}$-subgroup $M_{1}$ of $G$ which properly contains $M$. Hence $e \in M_{1}$, $e^{*} \in M_{1}^{*}$, and $M^{*}$ is necessarily a large modular $\Omega_{1}$-subgroup.

To prove that $M^{* \prime}=0$, suppose that $a^{*} \in M^{* \prime}$. Then $(a)^{*}=\left(a^{*}\right) \subseteq M^{*}$, so (a) $\subseteq M+M^{\prime}=M$. Thus $a \in M^{\prime}, a^{*}=0$ and the proof is finished.

Our final theorem is the following:

TheOREM 7. $A n\left(F, \Omega_{1}, \Omega\right)$-group $G \neq 0$ is $\Omega$-isomorphic to a subdirect sum of primitive $\left(F, \Omega_{1}, \Omega\right)$-groups if and only if $N=0$.

Let $G$ be $\Omega$-isomorphic to a subdirect sum of primitive $\left(F, \Omega_{1}, \Omega\right)$-groups $G_{i}$. This implies that $G$ contains a set of $\Omega$-subgroups $K_{i}$ such that $G_{i}$ is $\Omega$-isomorphic to $G-K_{i}$, and $\cap K_{i}=0$. Under the natural $\Omega$-homomorphism of $G$ onto $G_{i}$ with kernel $K_{i}, N$ maps into $N\left(G_{i}\right)$ which is zero since $G_{i}$ is primitive. Thus $N \subseteq \cap K_{i}=0$.

Conversely, if $N=0$, Theorem 6 shows that $\cap M_{i}^{\prime}=0$, where the $M_{i}$ are the large modular $\Omega_{1}$-subgroups of $G$. Thus $G$ is $\Omega$-isomorphic to a subdirect sum of the $\left(F, \Omega_{1}, \Omega\right)$-groups $G-M_{i}^{\prime}$, each of which is primitive by the lemma. This completes the proof of the theorem.

We conclude with some examples of the applications of the theory of this section. First, let $G$ be the additive group of a ring $R$, let $\Omega_{1}$ be the set of all right multiplications together with the identity automorphism, and $\Omega$ the elements of $\Omega_{1}$ together with all left multiplications. Thus an $\Omega_{1}$-subgroup of $G$ is a right ideal in $R$, and an $\Omega$-subgroup is an ideal. Let $F(a)$ be the set $\{a x-x ; x \in R\}$. In this case $N$ is the Jacobson radical of $R$, and $G$ is primitive if and only if $R$ is a primitive ring. If $R$ is taken to be a naring or a cluster, 
and we modify $F(a)$ to be the right ideal generated by the set $\{a x-x ; x \in R\}$, $N$ becomes the Jacobson radical of the naring or cluster [2].

Next, let $G$ be the additive group of the ring $R, \Omega$ the set of all right and left multiplications together with the identity automorphism, and $\Omega_{1}=\Omega$. Let $F(a)$ be an ideal such that under ring homomorphism $F\left(a^{*}\right)=F(a)^{*}$. By Theorem 4 we know that this implies property $\mathrm{P}_{1}$ which coincides with $\mathrm{P}$ in the present case. The ideal $N$ is now the $F$-radical of $R$. A large modular $\Omega_{1}$-subgroup is an ideal $M=M^{\prime}$ which, for some $a$ not in $M$, contains $F(a)$ and has the property that any ideal which properly contains $M$ also contains $a$. Thus $G$ is primitive if and only if $R$ is a subdirectly irreducible ring with zero $F$-radical $\left({ }^{4}\right)$. If, as a special case, $F(a)$ is chosen to be the (two-sided) ideal generated by the set $\{a x-x ; x \in R\}, N$ is the radical. A large modular $\Omega_{1}$-subgroup is a maximal ideal $M$ such that $R / M$ has a unit element, and $G$ is primitive if and only if $R$ is a simple ring with unit element. Again, if $R$ is taken as a naring instead of a ring, it is easy to modify these remarks so as to obtain the results of Smiley [10] on the F-radical or radical of a naring. More generally, the theory of the F-radical or radical of a cluster can be readily obtained in like manner.

\section{BIBLIOGRAPHY}

1. R. F. Arens and I. Kaplansky, Topological representation of algebras, Trans. Amer. Math. Soc. vol. 63 (1948) pp. 457-481.

2. B. Brown, An extension of the Jacobson radical, to appear in Proc. Amer. Math. Soc.

3. B. Brown and N. H. McCoy, Radicals and subdirect sums, Amer. J. Math. vol. 69 (1947) pp. $46-58$.

4. - The radical of a ring, Duke Math. J. vol. 15 (1948) pp. 495-499. 165-171.

5. - The maximal regular ideal of a ring, Proc. Amer. Math. Soc. vol. 1 (1950) pp.

6. R. A. Good, On the theory of clusters, Trans. Amer. Math. Soc. vol. 63 (1948) pp. 482-513.

7. N. Jacobson, The radical and semi-simplicity for arbitrary rings, Amer. J. Math. vol. 67 (1945) pp. 300-320. 430 .

8. R. Moufang, Zur Struktur von Alternativkörpern, Math. Ann. vol. 110 (1935) pp. 416-

9. M. F. Smiley, Alternative regular rings without nilpotent elements, Bull. Amer. Math. Soc. vol. 53 (1947) pp. 775-778.

10. - Application of a radical of Brown and McCoy to non-associative rings, Amer. J. Math. vol. 72 (1950) pp. 93-100.

11. M. Zorn, Theorie der Alternativen Ringe, Abh. Math. Sem. Hamburgischen Univ. vol. 8 (1930) pp. 123-147.

12. - Alternative rings and related questions I: Existence of the radical, Ann. of Math. (2) vol. 42 (1941) pp. 676-686.

Amherst College, Amherst, Mass.

Smith College, NoRthampton, Mass.

(4) See Theorem 5 of [3]. 